Fecha de recepción: marzo 2020 Fecha de aceptación: abril 2020 Versión final: junio 2020

\section{Dos miradas, dos ausencias. Estrategia y discurso en torno a la inasistencia de Daniel Scioli y Jair Bolsonaro a los Debates Presidenciales Televisados}

Carolina Verena Franco Häntzsch ${ }^{(1)}$

\begin{abstract}
Resumen: El 4 de octubre de 2015 se celebró el primer debate presidencial en la Argentina, organizado por Argentina Debate. El candidato del oficialismo, Daniel Scioli, decidió ausentarse una semana antes de la fecha pautada para la realización del duelo, elección inesperada que motivó la puesta en escena de un atril vacío durante la transmisión. Exactamente tres años más tarde, el 4 de octubre de 2018, excusándose con referencia a su salud, el entonces candidato Jair Bolsonaro no asistió al encuentro previo a la primera ronda de los comicios presidenciales en Brasil, parte de una tradición de debates ya consolidada en el país.

Partiendo de la base de que faltar a un debate presidencial televisado es una decisión costosa para un candidato en carrera, el presente artículo toma por objeto ambas ausencias. En primer lugar, explora hipótesis que expliquen la falta en términos de una opción estratégica. En segundo lugar, procede al examen de los efectos discursivos que la ausencia produce durante el desarrollo de las emisiones de debate. El análisis se sitúa en dos niveles: estratégico y discursivo. Las conclusiones conectan estas dos miradas y los hallazgos que ofrece el abordaje comparativo.
\end{abstract}

Palabras clave: Debates presidenciales - discurso político - campañas electorales.

[Resúmenes en inglés y portugués en las páginas 110 -111]

(1) Es Becaria Doctoral Conicet (Instituto de Investigaciones en Diversidad Cultural y Procesos del Cambio - Universidad Nacional de Río Negro), Doctoranda en Ciencia Política (Universidad Torcuato Di Tella), Lic. en Ciencias de la Comunicación (Facultad de Cs. Sociales, UBA). Participa de diversos proyectos de investigación en el cruce entre la comunicación y los medios y la ciencia política. Fue ayudante en la materia Semiótica de los Medios II (Fsoc - UBA) y docente en Política y Comunicación (UTDT). 


\section{Introducción}

En comunicación, la ausencia es siempre una jugada muy fuerte, y con un grado alto de incertidumbre. Lo importante es que la ausencia sea percibida por el o los destinatarios, no como resultado de factores externos, sino como una alternativa que el enunciador ha elegido entre otras alternativas posibles (Verón, 2014).

Aún en el entorno comunicacional y político contemporáneo, complejo, la televisión conserva un lugar central. Concentra miradas, alcanza audiencias lejanas, establece agenda de medios y redes sociales. En este contexto, los debates presidenciales televisados, acontecimientos mediáticos (Katz y Dayan, 1992) y políticos a un tiempo, son difíciles ignorar. Allí donde los debates presidenciales poseen cierta relevancia para la opinión pública, ausentarse puede ser una decisión costosa para un candidato en la carrera presidencial (Schroeder, 2000).

No obstante, si de algo están hechas las campañas presidenciales es de giros dramáticos e imprevistos. El 4 de octubre de 2015 se celebró el primer debate presidencial en la Argentina, organizado por la ONG Argentina Debate. El candidato del entonces oficialismo, Daniel Scioli, hizo pública la decisión de ausentarse una semana antes de la fecha prevista para la emisión. En el que hubiera sido su lugar, se conservó un atril vacío. El 4 de octubre de 2018, exactamente tres años más tarde, pero en Brasil, el entonces candidato Jair Bolsonaro no asistió al último encuentro anterior a la primera ronda de los comicios, organizado por Globo. Se excusó por referencia a su salud. En su lugar, mantuvo una entrevista exclusiva por la cadena Record, que fue transmitida en simultáneo.

Ocurre que, a pesar de las contraindicaciones, la evasiva entraña potenciales beneficios. Por más reglas y límites que equipos de campaña busquen oponer al libre desenvolvimiento del medio y de los contendientes, por más previsor que resulte su examen y el ensayo de sus movimientos, los debates representan una situación de riesgo para los candidatos (Jamieson y Birdsell, 1988; Schroeder, 2000; Milcovic, 2014). Acechados en simultáneo por el vivo y el directo y por la directa interpelación de periodistas y oponentes, los debatientes tienen mucho que perder al presentarse en el piso. La disyuntiva asistencia-ausencia, desde este punto de vista, constituye el núcleo de una serie de opciones estratégicas. Pero, como sugieren las palabras de Eliseo Verón, la ausencia también representa un acto enunciativo, que reverbera en imprevisibles consecuencias discursivas. Sin paradoja, el silencio de una ausencia dice y es dicho.

Nos interesa retomar aquí estas dos miradas: la ausencia como consecuencia, resultado de una decisión estratégica y la ausencia como causa, productora de efectos discursivos. Este doble abordaje responde en parte nuestra vocación exploratoria: desconocemos la existencia de estudios anteriores que hayan tomado a la ausencia de un candidato a un debate presidencial por objeto (quizás, por parecer escurridizo por definición). Al proponer una mirada integral sobre la negativa a presentarse a debatir, nuestro objetivo es iluminar el modo en que ciertos aspectos de la contienda política preelectoral se enlazan con lógicas comunicacionales y mediáticas. 
Concretamente, como adelantamos, este trabajo toma por objeto las ausencias de Jair Bolsonaro (PSL) y Daniel Scioli (FPV) a los debates presidenciales inmediatos anteriores a los respectivos comicios. Nos apoyamos en el registro grabado de ambas emisiones, y en fuentes secundarias. En Argentina, se trata del debate organizado el 4 de octubre de 2015 por la ONG Argentina Debate, en la Facultad de Derecho de la Universidad de Buenos Aires. Al encuentro asistieron todos los candidatos presidenciables, con excepción del oficialista Scioli: Nicolás del Caño (Frente de Izquierda) Adolfo Rodríguez Saá (Compromiso Federal), Sergio Massa (Frente Renovador), Margarita Stolbizer (Progresistas) y Mauricio Macri (Cambiemos), quien finalmente resultó electo, luego de una segunda ronda contra el candidato de FPV. Moderaron este debate los periodistas Marcelo Bonelli y Luis Novaresio, y lo condujo Rodolfo Barili.

En Brasil, nos referimos al último debate de la serie que diversos medios suelen realizar con anterioridad a las elecciones; el que nos ocupa fue organizado por la cadena Globo el día 4 de octubre de 2018, en Río de Janeiro. Fue conducido por William Bonner. Participaron Fernando Haddad (PT), Ciro Gomes (PDT), Geraldo Alckmin (PSDB), Marina Silva (Rede), Guilherme Boulos (PSOL) y Henrique Meireles (MDB). Como comentamos, Jair Bolsonaro, quien finalmente resultó electo tras una segunda vuelta contra Haddad, se ausentó argumentando la necesidad de extender su reposo tras el ataque sufrido el mes anterior. No obstante, mantuvo en simultáneo una entrevista en una cadena alternativa, Record. Por este motivo, consideramos igualmente pertinente evaluar la decisión de no concurrir desde un punto de vista estratégico.

Nuestro análisis sobre la inasistencia se extenderá en las dos direcciones sugeridas. Posando la mirada en las causas, en primer lugar, se explorarán hipótesis que expliquen la falta en su carácter de decisión táctica en el complejo entramado de estrategias que representa una campaña presidencial. Nos apoyaremos en fuentes secundarias y argumentos sugeridos por la literatura preexistente. Alternando el foco hacia los efectos, en segundo lugar, analizaremos las implicancias discursivas de la ausencia de los candidatos para el desarrollo de las respectivas emisiones. El examen del material comenzará por atender a la presentación indicial de la falta de ambas figuras a nivel de la puesta en escena, que entendemos que habilita a hacer inferencias acerca de la postura enunciativa que adopta la institución realizadora del debate. A continuación, indagaremos en las implicancias de esta ausencia para el desarrollo del duelo entre los candidatos presentes en el piso, con foco en la construcción adversarial del campo político (Verón, 1987) y en el desarrollo de los argumentos de los debatientes.

Finalmente, a partir de la lectura conjunta de los resultados de nuestra exploración en torno a la estrategia y de nuestro análisis discursivo, derivamos conclusiones con las que cerramos este artículo. Desde un punto de vista estratégico, encontramos que, para un candidato en carrera presidencial, ausentarse a un debate parece requerir como precondición gozar de cierta holgura en términos de intención de voto y popularidad. De todos modos, la falta debe apoyarse en una justificación que le otorgue sentido y coherencia, y la vuelva viable. Ahora bien, en la medida en que esté destinada a evitar que el candidato se convierta en el centro de los ataques, al observar sus efectos discursivos, encontramos que la evasiva no logra cumplir con su cometido. Independientemente de la postura que la institución organizadora adopte respecto de la ausencia, es decir, sea esta presentada y 
mostrada de manera expresa en pantalla o no, la falta se torna un argumento de peso en las estrategias de los debatientes. Los ausentes devienen en foco de la confrontación entre los presentes en el piso. Con todo, cerrando el círculo que plantea nuestro trabajo, encontramos que los blancos favoritos de la crítica no son los ausentes per se, sino las figuras que pueden encarnar de manera verosímil la representación de un mal inminente: estos son, los favoritos en la carrera electoral.

\section{¿Por qué faltaste? La ausencia: una estrategia}

Ya no es novedad decir que las campañas electorales, más aún las presidenciales, constituyen un terreno fértil sobre el que ha germinado toda una gama de expertos en comunicación: asesores, consultores, agencias de marketing y de relaciones públicas, encuestadores. Altamente profesionalizadas y planificadas (Norris, 2000; Vommaro, 2008), sería ingenuo atribuir las resoluciones de campaña al mero carácter o ideología de los aspirantes a los cargos públicos. Escapando a un tiempo a los absolutismos simétricos del voluntarismo y el determinismo, proponemos en este escrito examinar las decisiones de campaña de los actores políticos en términos de opciones estratégicas, adoptadas en los márgenes que permiten diversos condicionamientos.

Sobre este acuerdo de partida, nuestro trabajo propone explorar hipótesis que expliquen las ausencias de Bolsonaro y Scioli a los debates presidenciales televisados a la luz de los costos e incentivos que estos acontecimientos oponen a los candidatos. Si bien por principio similares, estos varían en cada elección y para cada potencial debatiente en función de aspectos que, a los fines expositivos, organizaremos en tres ejes. En primer lugar, destacaremos el peso del contexto político en el que se desenvuelve el candidato, puntualmente, su fortaleza y posición relativa en la carrera electoral. En segundo lugar, complementaremos esta mirada con una breve referencia al entorno mediático; dentro de este, en particular, nos interesa el estatus de los debates presidenciales en el país. De allí, en tercer lugar, pasaremos a la consideración de los dispositivos discursivos y comunicacionales en los que el aspirante apoya su campaña. Recurriremos, allí donde sea posible y pertinente, a trabajos y elaboraciones anteriores.

De acuerdo con el primer eje sugerido, la decisión de participar en un debate responde a la posición del candidato en el entorno político; particularmente relevante es el contexto electoral inmediato. Dos son las situaciones, expertos y asesores coinciden, bajo las cuales participar en un debate puede ser potencialmente pernicioso para un candidato en carrera: que este sea el "favorito", o que sea el candidato del partido en el gobierno. Invirtiendo la regla, las ventajas de asistir son sustancialmente mayores para los rezagados en la carrera electoral, y para los candidatos opositores, sobre todo los menos conocidos (Jamieson y Birdsell, 1988; Schroeder, 2000; Milcovic, 2014).

Resulta sencillo posicionar las ausencias de Daniel Scioli y Jair Bolsonaro a los respectivos debates presidenciales por referencia a estas presunciones. Comencemos por señalar que ambos candidatos llevaban la delantera en las encuestas al momento de realizarse el evento $^{1}$. No interesa a nuestros fines la precisión ni el grado de confianza con que estos 
informes hayan estimado los resultados a los que luego asistimos en los comicios. La clave radica en el peso de esta información para posicionar a un "triunfador" en agenda ${ }^{2}$. Amplificada y difundida por titulares y medios, las respectivas ventajas pasaron al repertorio del conocimiento público acerca de los pronósticos electorales. "Una brecha que se estira por arriba del diez por ciento. El sondeo de la consultora CEOP para Página 12, muestra un leve crecimiento de la fórmula del Frente para la Victoria, Scioli-Zannini, y una suave caída de Macri-Michetti”", titulaba y añadía a continuación el diario Página 12 unas semanas antes de la realización del primer debate presidencial, para ilustrar ${ }^{4}$. Similarmente, fueron frecuentes las noticias sobre el persistente aumento de la intención de voto hacia Bolsonaro en las semanas que antecedieron al encuentro televisivo que nos ocupa ${ }^{5}$.

En línea con las recomendaciones de expertos y asesores, en estas condiciones, presentarse a debatir entraña, en potencia, más riesgos que beneficios. En primer lugar, el candidato será previsible blanco de los contendientes, que buscarán sacar provecho de la oportunidad para acortar la distancia con el tenido como vencedor. En segundo lugar, cabe esperar que el desempeño del favorito sea el centro de la mirada mediática. En este sentido, cualquier desliz, furcio o desacierto promete resonar en innumerables repeticiones. Finalmente, la fama del candidato hace que este pueda prescindir del rating que asegura el acontecimiento. En vista de estas consideraciones, resulta razonable afirmar que tanto para Scioli como para Bolsonaro abstenerse de debatir era una opción estratégicamente coherente ${ }^{6}$. Retomando el peso de la posición política del candidato en la inasistencia al debate, al puesto en la carrera electoral cabe añadir su situación respecto del partido en el gobierno. En línea con las opiniones expertas que expusimos arriba, la creencia es que el oficialista ${ }^{7}$ posee ventajas respecto de sus competidores; por ende, es menos plausible que el debate le represente un beneficio. Cabe anotar que trabajos anteriores han aportado evidencia en favor del efecto positivo de la incumbencia sobre los resultados electorales. El diferencial consiste en el respaldo oficial y el acceso a recursos estatales de diverso orden en los que el aspirante puede apoyar su campaña ${ }^{8}$. Más aún, para el candidato del partido en el gobierno, debatir en televisión no sólo es innecesario, sino que conlleva mayores riesgos. Las razones que subyacen a este potencial perjuicio son similares a las que arriba planteamos que posee el favorito de en la carrera electoral (resumidamente, remiten al hecho de que su desempeño es intrínsecamente relevante tanto para el arco mediático, como para el político).

Esta variable aplica a nuestras figuras de interés de manera desigual. D. Scioli era el candidato oficialista, representante del kirchnerismo, entonces en control de la Presidencia. Ejercía el cargo de gobernador de la Provincia de Buenos Aires en nombre de esta fuerza política. La situación de J. Bolsonaro era diferente; a primera vista, casi inversa. El candidato provenía de un partido marginal a las fuerzas tradicionales y dominantes en el sistema brasileño ${ }^{9}$, y era ajeno tanto al Partido de los Trabajadores (PT), que había triunfado en las anteriores elecciones, de 2014, como al en aquél entonces compañero de fórmula MDB.

Ahora bien, no es fácil aplicar la aparentemente sencilla alternativa incumbencia-oposición a la situación en Brasil para las elecciones de 2018. Recordará el lector que el PT había sido removido del poder en 2016 mediante un impeachment a la entonces presidente D. Rousseff. Para las elecciones que nos ocupan, ostentaba el lugar de esta en el cargo el (ex) 
vicepresidente M. Temer (MDB), devenido en opositor al saliente PT. Buscaba sucederlo H. Meirelles, a quien podemos considerar el candidato oficialista en la campaña que nos ocupa. Por su parte, Bolsonaro y Temer no eran socios políticos, pero ambos habían sido parte activa en la destitución de Rousseff. En lo que concierne al PT, cabe agregar que el $1^{\circ}$ de septiembre de 2018, el Tribunal Superior Electoral resolvió rechazar la candidatura de I. Lula da Silva, su líder, preso desde el 7 de abril de ese mismo año, pero aprobó la coalición PT-PCdoB-PROS junto con la candidatura vicepresidencial de F. Haddad, quien fue finalmente designado candidato presidencial por el partido y la coalición diez días después. Dado este complejo entramado e inserción institucional de las diversas fuerzas políticas, la recomendación de que el incumbente no se presente al debate parece poco pertinente a los fines de comprender la situación brasileña.

Para continuar con el esquema sugerido, nuestro segundo eje corre la mirada del ámbito político al mediático. De los múltiples aspectos que se imbrican en los sistemas de medios a nivel nacional, con el fin de establecer los costos e incentivos que los candidatos poseen para presentarse a debatir, nos basta con atender al peso de la tradición de debates televisados en el país. Nos referimos al grado de institucionalización ${ }^{10}$ de esta práctica, y a su historia y relevancia a nivel local. Nuestra presunción es que cuanto más antiguos y consolidados son los debates presidenciales televisados, mayores costos enfrenta un debatiente para rechazar la invitación (encontramos sugerencias que apuntan en esta dirección en Schroeder, 2000).

Entre los casos que examinamos, de nuevo, la diferencia es sustancial. Brasil se destaca por ser el primer país latinoamericano en haber organizado debates presidenciales televisados (Ruiz y Alberro, 2012). La práctica sobrevivió, a su manera, bajo los años autoritarios. Desde la recuperación de la democracia, se han celebrado debates para todas las elecciones presidenciales. Aunque no son obligatorios, existen prescripciones formales instituidas en la ley electoral del país desde 2012. En marcado contraste, en Argentina se considera que el primer debate presidencial televisado organizado con éxito fue, precisamente, el que nos ocupa: al que Scioli decidió ausentarse, en 2015. Si bien en ciertos distritos se habían practicado debates de manera previa a otras instancias electivas, a nivel presidencial los intentos hasta entonces habían sido infructuosos (Acosta, 2016; Quirós, 2015; Slimovich, 2011; Waisbord, 1995). Dada esta disparidad entre Brasil y Argentina, es difícil atribuir un peso determinante a esta variable sobre las decisiones de Bolsonaro y Scioli de faltar a los respectivos duelos televisivos. Sin embargo, no descontamos que la baja institucionalización de la práctica haya facilitado la inasistencia de este último al encuentro.

Con todo, podemos complejizar nuestra lectura de la dimensión mediática si tomamos en consideración que el poder de los medios en la política es en buena medida un poder reputacional (Kitzberger, 2016). Desde esta perspectiva, las percepciones de los candidatos acerca de los riesgos en los que incurren al "descuidar" su relación con los actores de medios (en este caso, a partir de la negativa a concurrir a un debate) se nutren de experiencias anteriores. En esta línea, hallamos a ambos lados de la frontera situaciones pasadas, de público conocimiento, en las que un candidato presidenciable había decidido ausentarse a un debate provocando amplio rechazo del arco mediático, y, no obstante, ganado las elecciones. 
En Argentina, el "faltazo" tuvo a C. Menem (Partido Justicialista) como protagonista. Para las elecciones de 1989, había sido invitado a debatir en televisión contra su principal opositor, E. Angeloz (Unión Cívica Radical), en el programa Tiempo Nuevo, bajo la dirección del periodista B. Neustadt. La ausencia de Menem fue simbolizada con una silla vacía en el estudio (técnicamente, entonces, no hubo debate alguno, ya que el encuentro estaba planeado para estos únicos dos contendientes). Menem resultó presidente electo en los comicios que se celebraron 5 días después, con 47,5\% de los votos. En Brasil, fue destacada la inasistencia de Lula da Silva a la totalidad de los encuentros organizados para las elecciones de 2006, en las que el entonces presidente buscaba la reelección. A pesar de la crítica pública, los resultados ratificaron a Lula en el cargo, tras una segunda vuelta contra el primer opositor, G. Alckmin. No buscamos evaluar la relevancia o irrelevancia de la crítica mediática vis-a-vis otros factores sobre los resultados electorales. Antes bien, con base en estos relatos, sugerimos que tanto para Bolsonaro como para Scioli existían referencias anteriores que indicaban que ausentarse a un debate, aún a riesgo de despertar numerosas críticas, no era en absoluto determinante para su desempeño electoral.

Un aspecto ulterior de la dimensión mediática que puede resultar pertinente examinar remite al paralelismo político de los medios de comunicación (Hallin y Mancini, 2004); más precisamente, a la medida en que estos son percibidos como actores con intereses y poder en el juego político. En breve, entendemos que la legitimidad de los debates se erige sobre la pretensión de que constituyen arenas de discusión neutral y estrictamente regulada (Jamieson y Birdsell, 1988). Allí donde esta objetividad se crea imposible, los debates televisados pueden ser impugnados (se sospechará que son meros instrumentos de campaña, favorables a determinados intereses o fuerzas políticas). Precisamente, en los dos casos que interesan a este escrito, la inasistencia se enlaza en un contexto de cuestionamientos expresos e implícitos al rol neutral y mediador de los medios de comunicación. Para profundizar en esta lectura consideramos pertinente introducirnos en el tercer eje que esquematizamos anteriormente: el de las estrategias comunicacionales y discursivas de los respectivos candidatos. Desde aquí, esperamos complementar la mirada acerca de los riesgos e incentivos que enfrenta un candidato al ausentarse a un debate, con el análisis de cómo la ausencia se imbrica en una narrativa de campaña específica, que hace un uso particular de los múltiples dispositivos para la comunicación política que los candidatos tienen a su disposición. Así, esperamos comprender el modo en que la ausencia pudo ser justificada, terciando de la lógica de costos y beneficios que planteamos hasta aquí.

Comencemos por Daniel Scioli. Como hemos elaborado en un trabajo anterior (Franco, en prensa), su inasistencia al debate presidencial puede ser comprendida como parte de una estrategia comunicacional y discursiva más amplia, que hace a la relación que el kirchnerismo mantuvo con los medios de comunicación. Las prácticas comunicacionales del kirchnerismo se han definido por una confrontación dirigida hacia el sistema mediático en general, $y$, dentro de éste, hacia determinados actores en particular. El origen de esta disputa, o su exacerbación, se remonta conflicto que enfrentó a la entonces gestión y sectores de la producción agraria en $2008^{11}$, punto de inflexión para la radicalización política, discursiva y comunicacional de esta fuerza política (Becerra, 2008; Kitzberger, 2011; Raiter, 2013). Se han relevado tres dimensiones interrelacionadas desde las que se gestó el conflicto: la regulatoria, la comunicacional, y la discursiva (De Diego, 2014; Kitzberger, 
$2016)^{12}$. En pocas palabras, se ha teorizado que esta confrontación a múltiples niveles entre medios y kirchnerismo extraña "una disputa por el contacto con los ciudadanos" (Fernández, 2014: 35), "una disputa por la legitimidad de la representación" (De Diego, 2014: 8). En este contexto, la negativa de Scioli a participar en el debate se inscribe en una disputa por la legitimidad que los medios poseen para constituirse en mediadores pretendidamente neutrales entre la ciudadanía y sus gobernantes. De modo complementario, se entrama en una estrategia inclinada a establecer modos de comunicación "directos" con el público. Los argumentos que la prensa recoge con respecto a su inasistencia ratifican ambas lecturas: “Mi plan está claro, lo demás es chamuyo' (...) El gobernador bonaerense afirmó que 'la gente sabe muy bien' qué es lo que él piensa”, relataba una nota de Clarín de octubre de $2015^{13}$. Otra nota en Infobae recogía declaraciones "de un dirigente de la ola naranja", que apuntaba que "esto genera un tironeo mediático y te expone a operaciones políticas que no suman"14. En suma, la justificación públicamente esgrimida de la falta es coherente con las propuestas discursivas y comunicacionales de su fuerza política: Scioli esperaba interpelar al electorado por fuera de las mediaciones periodísticas, que entendía como sesgadas, "políticas".

Similar lectura podemos aplicar al caso de J. Bolsonaro, si bien desde otro lugar. En cuanto a sus dispositivos comunicacionales de preferencia, se ha destacado el importante rol que cumplieron redes sociales en el despliegue de su campaña. El candidato convocó apoyos mediante plataformas digitales; de manera destacada, a través de su perfil en Facebook (Anderson, 2019). En consecuencia, contaba con su habilidad para suscitar apoyos de manera "directa", por fuera de los dispositivos "tradicionales" (entre ellos, los medios masivos de comunicación). Desde la óptica de las elaboraciones discursivas, el candidato abrevó de similar impugnación a la presunta objetividad de periodistas y de ciertos actores de medios específicos. Aún sin analizar en detalle su argumento, hemos registrado que la prensa y la opinión pública en general se han hecho eco de su referencia a la noción de "fake news", a tono con ciertas preocupaciones contemporáneas. De este modo, espejando la situación de su par argentino, la campaña de Bolsonaro se desenvolvía entre dispositivos comunicacionales y elaboraciones discursivas que confrontaban con la prensa como lugar de interpelación a la ciudadanía. En consecuencia, faltar al debate era una opción viable y coherente.

Nuestra mirada acerca de las justificaciones que rodean al caso brasileño estaría incompleta si no hiciéramos referencia al incidente que tuvo a Bolsonaro como protagonista durante la convulsionada carrera electoral: el atentado que sufrió el candidato el 6 de septiembre durante un evento de campaña en Minas Gerais. De hecho, Bolsonaro se ausenta a todos los debates presidenciales a partir de ese momento ${ }^{15}$. Durante la primera semana estuvo en recuperación en el hospital; igualmente, luego de su salida, se mantuvo la dinámica de las inasistencias. Entendemos que la eventualmente delicada situación de su salud durante los primeros días de septiembre no logra objetar el análisis respecto de su inasistencia al último encuentro televisivo, ya que el candidato decidió "competir" contra esta emisión ofreciendo una entrevista exclusiva en la cadena Record. En todo caso, agrega a las consideraciones elaboradas hasta el momento un argumento de peso para inscribir y legitimar la inasistencia: la "fuerza mayor"16. Scioli, por su parte, carecía de justificativo semejante. Hemos explorado los tres ejes propuestos. Con base en lo recorrido hasta aquí, podemos 
adelantar algunas conclusiones. En vista de las diferencias y similitudes halladas en los condicionamientos presumiblemente relevantes a la hora de explicar las inasistencias de los candidatos que nos ocupan a los debates, sobresale el peso de la delantera en la carrera electoral. De mínima, podemos afirmar que un aspirante a presidente necesita suponer que cuenta con cierta ventaja electoral para afrontar los costos de faltar a debatir. De manera complementaria, la viabilidad de la negativa parece requerir su inscripción coherente en una trama comunicacional y discursiva que la justifique y le dé sentido. En contraste, otros factores históricos y contextuales, políticos y mediáticos, difieren entre los casos que nos ocupan, $y$, por ende, parecen menos determinantes en la decisión: la relación del candidato con el partido de Gobierno, la escasa institucionalización de los debates en el país, o, alternativamente, la posibilidad de esgrimir un argumento de "fuerza mayor" que resulte verosímil. Con todo, no descontamos que estos factores se constituyan en incentivos adicionales, que cooperen en promover la falta. Hemos argumentado además el eventual peso de experiencias pasadas en la percepción de los candidatos acerca del balance de costos y beneficios que afrontarán al inasistir a un debate televisado. En cualquier caso, una vez resuelta la ausencia, de esta se desprenden consecuencias escapan a su control. Concentraremos nuestra atención en ellas en el apartado a continuación.

\section{La ausencia, presente}

Si hasta ahora nuestro examen se detuvo en las causas, en lo que sigue posaremos nuestra mirada en los efectos de las respectivas ausencias, en un nivel estrictamente discursivo. Tomaremos por objeto ambas emisiones de debate presidencial. En primer lugar, observaremos las representaciones de la ausencia a nivel escenográfico, desde donde indagaremos la postura enunciativa institucional de los organizadores de cada debate. En segundo lugar, investigaremos el rol de la ausencia en las argumentaciones de los candidatos presentes, con particular foco en la construcción adversarial del campo político (Verón, 1987). Para ambas tareas, tal como hemos hecho hasta aquí, esperamos sacar provecho de la mirada comparativa.

De acuerdo con el recorrido propuesto, comencemos por analizar las presentaciones de la ausencia en el nivel escenográfico. Los modos de significar en este orden reenvían de manera saliente a la indicialidad: las relaciones existentes entre los cuerpos, la espacialidad, los movimientos (Verón, 1993). A este respecto, las repercusiones de la falta de Scioli y de Bolsonaro a las respectivas emisiones varían.

La puesta en escena en el caso brasileño disimula la inasistencia. Los candidatos esperan su turno para debatir sentados en los laterales de la pantalla. A la hora de hacer preguntas o de ser designados para ofrecer respuestas, deben pararse en una suerte de "arena" central. 


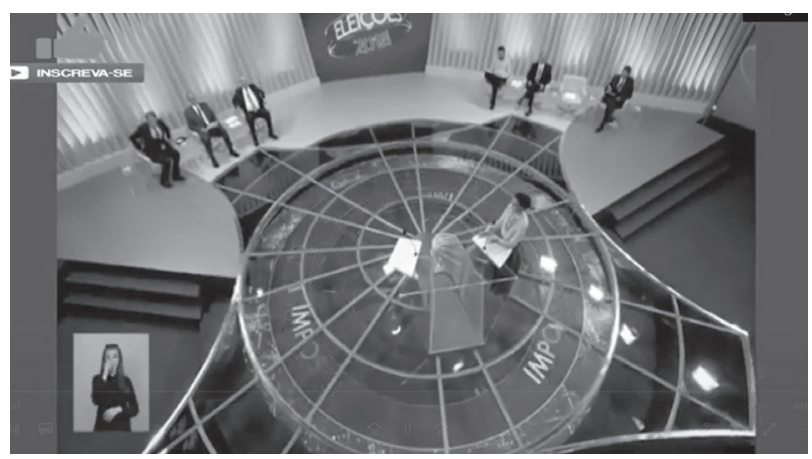

Figura 1. La puesta en escena del debate de la cadena Globo disimula la ausencia del candidato J. Bolsonaro. Fuente: https://g1.globo.com/ politica/eleicoes/2018/ noticia/2018/10/05/veja-aintegra-do-debate-na-globo ghtml

Un observador muy incisivo podría atender a un desbalance menor: a un lado de la pantalla hay tres candidatos; al otro, cuatro. Por lo demás, el diseño escenográfico, sobrio y sencillo, no señala en pantalla el espacio de un cuerpo ausente.

En contraste, en el caso argentino, la inscripción de la ausencia del cuerpo de Scioli es expresa y evidente. Dispuesto entre los candidatos presentes en el piso, el espectador asiste a un atril vacío. A esto se añade, a tono con la grandilocuencia de la escenografía, una pantalla detrás de cada debatiente, que amplifica la representación del contrapunto ausencia-presencia a partir de la mostración de los rostros de los asistentes, por un lado, y las diversas gráficas institucionales, por el otro.

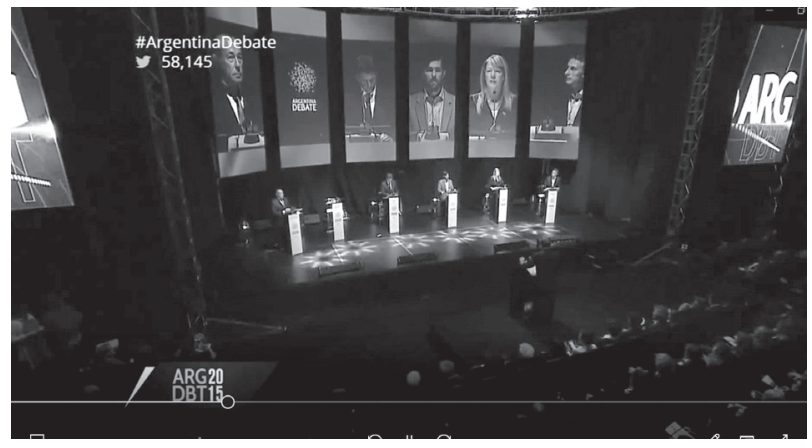

Figura 2. En el primer debate organizado por Argentina Debate en 2015, la falta de D. Scioli es presentada a partir de la colocación de un atril vacío en la escenografía, delante de su respectiva pantalla. Fuente: https:// www.youtube.com/ watch?v=bkK8iI2qZVg 
Aún en este nivel indicial, la falta de Scioli se completa a partir de la inscripción de su silencio. Organizadores del debate repartieron entre los debatientes el tiempo de exposición originalmente reservado al inasistente ${ }^{17}$. Reenviando a una respuesta imposible, con modalidad subjuntiva, los candidatos aprovecharon el espacio para formular hipotéticas interrogaciones: "a Daniel Scioli me hubiese gustado preguntarle, cómo cree que la educación es una prioridad, si así fuese para él..." (N. del Caño, AD $)^{18}$. En el caso que más refuerza la idea de vacío, un contendiente hizo expreso el silencio: luego de argumentar que "lo mejor que podemos hacer es que su silencio, que es una burla a la sociedad, quede plasmado" (AD), S. Massa permaneció mudo la totalidad de su turno. En contraste, el formato brasileño no contempló semejante readjudicación de los tiempos de exposición. Se mantienen así las diferencias que encontramos a nivel de la puesta en escena entre los dos debates que nos ocupan.

Puede entenderse que la puesta en escena y el formato de los intercambios que examinamos aquí constituyen una toma de posición por parte del organizador institucional de los debates. En otras palabras, escenografía y formato remiten a un enunciador institucional cuyas disposiciones organizan, encauzan, el despliegue de la indicialidad durante el desarrollo de las emisiones. Desde esta mirada, con base en lo expuesto hasta aquí, tenderíamos a concluir que Bolsonaro obtuvo un trato imparcial, mientras que la invocación de la falta de Scioli edifica una postura crítica. Ahora bien, de manera complementaria, en un nivel simbólico, este enunciador institucional también aparece delineado en la voz de los moderadores; esto es, de los periodistas que arbitraron el encuentro en nombre de la institución. Examinar el modo en que refieren a las ausencias habilita lecturas alternativas. Tanto en el debate brasileño como en el argentino, los conductores mencionan a los respectivos inasistentes. En ambos, las referencias son breves, estrictamente informativas, y entrañan una suerte de "respeto" a los motivos formulados por el candidato que no concurrió a debatir. En el debate de la cadena Globo, la única mención de Bolsonaro por parte de W. Bonner es mínima: "El candidato Jair Bolsonaro, del PSL, también fue invitado, pero informó que no podría comparecer porque todavía se recupera del atentado que sufrió el día 6 de septiembre" (G). Este único comentario, en el inicio de la transmisión, introduce la justificación que el propio inasistente había esgrimido. En el debate organizado por Argentina Debate, la dinámica es similar, aunque hallamos algunas diferencias: destacamos que, si bien breves, las referencias al candidato ausente son reiteradas. En cualquier caso, de manera afín a como recién señalamos para su par brasileño, los moderadores se encargan de reponer las explicaciones que el propio Scioli ofreció sobre su ausencia. Así, mediante discurso referido indirecto M. Bonelli comenta: "lo esperábamos a Daniel Scioli que no ha concurrido; él previamente ha dicho que no iba a venir, por eso el atril que está vacío" (AD).

Además, tanto el moderador brasileño como los argentinos expresan y reiteran que el formato del debate y su propio papel responden a reglas predeterminadas y consensuadas. Las intervenciones de Bonner son escasas: explica la modalidad de los intercambios, ordena la secuencia de las exposiciones, pide silencio, muestra en vivo y alude verbalmente el peso de los sorteos en la determinación de la dinámica del evento. En Argentina Debate, el comportamiento de los moderadores responde a similares funciones. En esta línea de argumentos, los conductores explicitan que el equipo de campaña de Scioli fue parte de los 
acuerdos que antecedieron a la realización del evento: "como decía Marcelo, hay muchas reglas (...) Todas y cada una de las reglas fueron acordadas por los seis equipos de candidatos. Están suscriptas, firmadas" (L. Novaresio, AD) ${ }^{19}$.

De lo expuesto, se infiere que la toma de posición del enunciador institucional a nivel verbal, en la voz que corporizan moderadores, entraña la expresa búsqueda de imparcialidad, de agente actúa conforme a reglas. Esta toma de posición neutral puede aparecerse como más o menos ambigua en conjunción con las decisiones enunciativas que la institución pone de manifiesto a nivel de la puesta en escena, que repusimos con anterioridad. Con todo, lo que interesa destacar es la aparente necesidad del medio de erigirse en arena neutral en aras de garantizar la legitimidad del evento. Dicho al revés, en ambos debates el enunciador-realizador no quiere, o no puede, manifestar sesgo u opinión.

La pretensión de objetividad del enunciador institucional contrasta con el lugar central de la falta en la polémica que entablan los debatientes: ésta se erige en polo de la confrontación. Estamos aludiendo aquí a la segunda línea de efectos discursivos de la ausencia que propusimos examinar: su peso en la construcción adversarial del campo político. Examinaremos esta dinámica en lo que sigue. Encontraremos, para ambos casos, que la disputa toma la forma de un eje "nosotros (presentes) - ellos (ausentes)". No obstante, indicaremos algunas diferencias pertinentes entre las situaciones de debate argentino y brasileña. Con base en el examen comparativo, hallaremos que la figura del ausente deviene relevante para la argumentación y la polémica en un debate presidencial en la medida en que sintetiza ciertas funciones, que se derivan de su posición en el entramado político tal como lo definimos en el apartado anterior: su puesto en la carrera electoral, y su relación con el partido de gobierno. Inversamente, podemos decir que el favorito, el oficialista, y el ausente son los roles que se erigen en el blanco preferido por los debatientes. En Argentina Debate, Scioli condensa estas tres funciones, dando lugar a una estructuración lineal de la polémica, que toma la forma de "todos, nosotros" versus "ellos, el kirchnerismo". En Brasil, la situación es más compleja. La intrincada situación política posiciona de manera alternativa en los roles del ausente, del favorito y del oficial a J. Bolsonaro, I. Lula da Silva y M. Temer. En consecuencia, múltiples ejes conflictivos se entraman en este debate. Prosigamos a su análisis.

Comencemos por el caso argentino, que ya hemos analizado en un trabajo anterior (Franco, en prensa). En primer lugar, como adelantamos, los candidatos presentes critican explícitamente a D. Scioli. En más de una ocasión, argumentan con base en su decisión a faltar al debate (si bien el abanico de críticas no se agota allí). Para ilustrar, observemos el siguiente extracto de una respuesta de M. Stolbizer a A. Rodríguez Saá. En este, de la ausencia de Scioli, una acción, se deriva la carencia de una capacidad que se presupone como necesaria a los fines de gobernar. Retengamos, además, el uso del nosotros inclusivo, que aúna a la candidata y su interlocutor, con quien la búsqueda de complicidad es reforzada por el discurso referido a un momento de diálogo anterior ("de los que hablábamos"): "creo que es muy importante trabajar en esa construcción de los consensos de los que hablábamos, porque además el que no vino a debatir no tendrá la capacidad para construir ese consenso" (AD).

En segundo lugar, la crítica apunta al kirchnerismo como fuerza política. Detectamos dos modalidades principales. Por un lado, las referencias expresas o alusiones en las que se 
lo asocia con actitudes o cualidades negativas. Por ejemplo, el comentario de M. Macri, en el que de la comparación se deriva un énfasis argumentativo en lo evidente: "yo hace ocho años que gobierno la Ciudad de Buenos Aires; en ocho años, no tuvimos una denuncia por corrupción, en los últimos veinte días, decenas: obviamente, es la forma de hacer campaña que tiene el kirchnerismo" (AD). Por otro lado, la crítica hacia el kirchnerismo se oficia a partir del comentario impersonal que identifica problemas que, por implicación, quedan asociados a la gestión del Estado, y, por ende, al partido en control del gobierno; sea en el nivel nacional o subnacional. Por ejemplo, S. Massa hace un uso frecuente del impersonal para referir a las causas de situaciones consideradas como problemáticas, dejando al espectador la tarea de completar la referencia: "la plata del sistema jubilatorio, que la ley 26.111 establece de distribución por sistema solidario, está siendo usada para otra cosa" (AD). O bien, para ilustrar, la frase de A. Rodríguez Saá ancla territorialmente un problema a la Provincia de Buenos Aires, bajo gestión de D. Scioli: "la Provincia de Buenos Aires tiene un déficit enorme: avanzan los asentamientos y las villas de emergencia" (AD).

En breve, entonces, hallamos una relación parte-todo que conlleva la extensión de la crítica desde Scioli hacia el kirchnerismo, y viceversa. De este modo, el candidato y su fuerza política quedan constituidos en el principal polo de la confrontación en las argumentaciones de los debatientes. Además, hemos notado que los candidatos utilizan el nosotros inclusivo, que tiene el efecto de ubicar a los supuestos contrincantes en el polo de la prodestinación. En esta línea, en la apretada síntesis de la dinámica polémica del debate argentino que ofrecemos aquí, subrayamos el carácter cuasi amistoso, cortés, que caracteriza a los intercambios entre los presentes en el piso. En voz de los candidatos aparecen referencias expresas a la búsqueda de complicidad y de lugares comunes compartidos, y a campos semánticos que remiten a las nociones de acuerdo, de consenso, de diálogo, y de trabajo conjunto, junto al uso del nosotros inclusivo que comentamos en lo anterior $^{20}$. Hemos ilustrado esta operatoria con la frase de M. Stolbizer; por motivos de espacio, no nos extendemos en ejemplos adicionales aquí. En síntesis, el primer debate presidencial argentino se caracteriza por una dinámica amistosa entre los presentes en el piso, desde donde se erige una confrontación expresa y conjunta contra el candidato ausente y el colectivo de identificación en el que se lo sitúa: nosotros versus el kirchnerismo. En el debate brasileño, como adelantamos, hallamos una lógica confrontativa similar, pero con una trama más compleja. Al igual que Scioli para el caso argentino, Bolsonaro, inasistente, se erige en polo de la polémica. Ahora bien, los debatientes entablan conflictos con otras dos figuras igualmente ausentes: M. Temer e I. Lula da Silva; este último, bajo el paraguas del PT. De este modo, hay tres adversarios privilegiados, tres blancos de las críticas de los candidatos en el piso, que estructuran tres ejes de conflicto, e inscriben la complejidad de la coyuntura política brasileña en el desenvolvimiento argumentativo de la emisión. Analizaremos este entrecruzamiento de lógicas conflictivas en lo que sigue, con apoyo de fragmentos que ilustran su despliegue.

Comencemos por la figura que convoca a este escrito: J. Bolsonaro. Como decíamos, de manera análoga a su par argentino, el candidato ausente se erige en blanco privilegiado de los presentes en el estudio de la cadena Globo. De manera igualmente semejante a lo ocurrido con D. Scioli, a Bolsonaro no sólo se lo critica por su ausencia. Su carácter, 
sus acciones y sus palabras son referidos en la confrontación. No obstante, y a pesar de que el formato y el moderador de este debate no la convocan expresamente, la falta es instrumentada como parte de la evidencia argumentativa. De manera similar a ciertos argumentos examinados para el caso argentino, el razonamiento parte de la acción concreta y generaliza hacia la incapacidad del inasistente para responder a responsabilidades y tareas que se presuponen como relevantes para el cargo de la Presidencia.

Por ejemplo, en el siguiente intercambio, C. Gomes y H. Meirelles hacen una lectura moral de la inasistencia en términos de lo que se considera correcto-incorrecto para "un hombre que quiere ser presidente". Como observamos en la respuesta de Meirelles, se infiere que un candidato que no concurre a debatir es un candidato que no responde a la soberanía del electorado, cuya importancia es tenida como premisa. Subrayamos además que este supuesto compartido despeja el camino para un intercambio cordial entre ambos candidatos, en el que se repiten las muestras de asentimiento y de mutuo acuerdo. Notemos en particular las frases introductorias que retoman y afirman el argumento precedente, con base en el uso de deícticos: "eso está mal", "es importante eso".

C. Gomes: -Meirelles, me gustaría hacer esa pregunta que le iba a hacer al candidato Bolsonaro. (...) él hoy está de alta y da una entrevista larga, mucho más extensa de lo que nosotros estamos conversando aquí, cada uno de nosotros va a hablar diez minutos. Se escapó. ¿Halla usted correcto que un hombre que quiere ser presidente de Brasil no se someta a debate?

H. Meirelles: -No, eso está mal. Y muestra a alguien que no sólo está fugándose del debate, sino de su compromiso con la población. Y, más importante, no es meramente el debate, es el estar aquí sometido a crítica (...), cada uno de nosotros está aquí, enfrentando eso con seriedad y con respeto al elector. Y esto es lo que es importante, el elector merece respeto (...).

C. Gomes: -Es importante eso, brasileño. El candidato que lidera las encuestas (...), cuando ve la repercusión de [las medidas que anuncia su vice], se las niega a la prensa. Aquí tenemos (...) una mentira, que requería que él estuviera aquí para que la gente pueda esclarecerla (...). (G)

Agreguemos un ejemplo adicional de la crítica explícita hacia la falta, ya que nos presenta una estrategia argumentativa reiterada a lo largo del debate en voz de distintos candidatos. Esta consiste en asociar figuras ausentes y presentes con el objetivo de diferenciarse de ambas a la vez; adoptando bien la posición de simple opuesto a un contradestinatario global o englobante, o bien la de un tercero cuya postura se delinea por contraste con dos alternativas igualmente negativas -en breve, la confrontación se visualiza como una línea, o como un triángulo-. En el fragmento a continuación, esta última estrategia es utilizada por M. Silva. Observemos que, tal como hacían participantes del debate argentino, la candidata expresa con modalidad subjuntiva su deseo de elaborar preguntas al ausente, convocando, trayendo a presencia, su falta. A partir de allí, la candidata presenta un panorama con dos polos polémicos, en el que luego podrá posicionarse como tercera opción. En este caso, se trata de Bolsonaro y el PT; este último incluye a F. Haddad, su interlocutor en el debate: 
Yo le haría esa pregunta al candidato Bolsonaro, pero como él una vez más se acobardó, dio una entrevista para Record y no vino aquí a debatir con nosotros. Tenemos hoy una situación en la que el $25 \%$ está votando porque no quiere a Bolsonaro, $25 \%$ vota porque no quiere al candidato del $\mathrm{PT}$, que es usted. $50 \%$ no quiere a ninguno de los dos. $(\mathrm{G})$

En efecto, el PT se erige, como decíamos, en el segundo adversario privilegiado de la contienda. Es el contradestinatario en una serie de argumentos que tienen a la gestión del gobierno como eje. El funcionamiento es afín al que posicionaba al kirchnerismo como polo de la confrontación en el caso argentino. No obstante, como el lector podrá inferir, en este caso, la forma argumentativa parte-todo no convoca a Bolsonaro, sino que permite englobar al candidato presente, Haddad, e invita a una segunda figura ausente: Lula da Silva. Observemos dos fragmentos que ilustran esta serie de constructos argumentativos. En el primero, A. Días y G. Alckmin, de manera similar a como hacían Gomes y Meirelles en el caso anterior, apoyan un intercambio cordial sobre la base del mutuo acuerdo: la crítica compartida hacia el PT. Por añadidura, ambos refuerzan sus propias posiciones buscando diferenciarse de otros polos en la confrontación. En Dias, en forma de un taxativo "fui oposición la vida entera", que enuncia en primera persona. En Alckmin, a partir de la identificación de dos polos polémicos, que actualizan sin nombrarlos al PT y a Bolsonaro, y los aúna y asimila bajo la idea de radicalización. Establece de este modo una estrategia de confrontación "triangular", tal como introducimos recién.

A. Dias: -Geraldo Alckmin. Geraldo, yo te respeto mucho, y ahora, más de una vez aquí, se confirmó que, en la olimpíada de la mentira, el PT gana una medalla de oro. Constantemente se afirman cosas que no acontecieron, por ejemplo, que yo apoyé esto, apoyé aquello. Yo fui oposición la vida entera. (...) ¿Cuál es tu propuesta para cambiar esta realidad del país, el modelo que está vigente?

G. Alckmin: -Oiga, yo creo que nosotros ya tuvimos, Alvaro Dias, ya tenemos (...) una larga experiencia del PT y vimos el resultado de eso. El resultado fueron 13 millones de desempleados, criminalidad en alza (...). Ahora, también del otro lado, y yo vengo hace meses hablando de esto, el camino tampoco es un radical de derecha, que no tiene la menor sensibilidad, ¿entiende? (...) Entonces, nosotros que siempre estamos en un camino diferente que los radicales de derecha y de izquierda tenemos que, en estos tres días, llevar a una reflexión profunda a Brasil. No podemos ir hacia esa segunda vuelta entre extremos. $(\mathrm{G})$

En el segundo ejemplo, destacamos el modo en que Lula, ausente, es invocado de manera polémica en el piso por A. Dias, operación que este debatiente reitera en más de una ocasión. Observemos que el PT en posición de contradestinación reúne a Haddad y a Lula. Además, el candidato aprovecha la referencia a este polo polémico, parcialmente ausente, para introducir lo que presupone una premisa compartida con su interlocutor, Meirelles; esta es, que los problemas contemporáneos tienen su origen en la corrupción. 
Oiga, traje una pregunta por escrito y se la voy a entregar, al final, a Haddad, para que él la lleve al candidato del PT, que está preso en Curitiba, ya que lo visita todos los lunes. Y le quiero decir a Meirelles que estamos discutiendo mucho en esta campaña electoral que es periférico. Estamos dejando de discutir lo que es esencial, el modelo que nos gobierna. Ese modelo corrupto, incompetente. $(\mathrm{G})$

Cabe añadir que, de manera simétrica e inversa, la asociación con Lula y el PT es también invocada en un sentido positivo, de refuerzo a la propia posición, en voz del candidato del partido, Haddad. Observemos esta dinámica en el breve fragmento a continuación. El candidato expresamente convoca la figura del ausente, que asocia a un proyecto del que se coloca como representante.

Oiga, Marina, yo agradezco su pregunta. Yo estoy en campaña hace apenas 22 días. Entré en una situación completamente anormal. El líder de las encuestas, que figuraba en la delantera, pudiendo ganar en el primer turno, no puede participar de la elección en función de una decisión arbitraria (...). Usted sabe de quién estoy hablando. Estoy hablando de Lula, que tenía $40 \%$ de intención de voto. Yo me estoy presentando al electorado porque represento en este momento un proyecto que funcionó. $(\mathrm{G})$

Como contrapunto, a lo largo del debate este candidato introduce la tercera ausencia que hallamos en la dinámica conflictiva que nos ocupa: M. Temer. Atendamos al siguiente fragmento ilustrativo, que da cuenta de esta tercera presencia ausente en las disputas entre los participantes brasileños. En esta ocasión, el candidato del PT abreva de la figura de Temer para criticar a su contendiente, Alckmin. El acusado se defiende a partir de una inversión de la crítica. Busca, bajo el concepto de responsabilidad, asociar al PT con Temer en un mismo polo confrontativo, en el que reúne figuras ausentes y presentes a las que se opone linealmente. En esta dinámica, a fin de cuentas, Haddad y Alckmin comparten la presunción de que la figura de Temer es negativa. Redondeando nuestro argumento, observemos además que Alckmin expresamente introduce las tres figuras polémicas reiteradas a lo largo del debate (en voz de los diversos contrincantes): añade sobre el final una referencia a Bolsonaro, a quien posiciona en plano de equivalencia con el PT por referencia a su incapacidad.

F. Haddad: -(...) Lo que el candidato Geraldo Alckmin no reconoce es que después de que su partido fue derrotado en 2014 (...), el PSDB se asoció a Michel Temer para sabotear al gobierno, aprovechando las llamadas pautas-bombas (...). Y fue eso lo que llevó el país a la crisis. Y no la política responsable con las finanzas públicas que nosotros llevamos adelante.

G. Alckmin: -Oiga, estamos en completo desacuerdo. Yo quiero hablar con usted que nos está escuchando. El PT terceriza responsabilidades. El PSDB está fuera del gobierno desde hace 16 años. (...) Tenemos... quién escogió a Temer fue el PT, fue Dilma. Cuando dijo que iba a pactar con el diablo para ganar 
la elección. Ganar la elección dando un golpe al elector, porque hizo al país ingobernable. Yo no creo que PT, tampoco Bolsonaro, vayan a sacar a Brasil de la crisis (...). (G)

De camino a cerrar este recorrido por la estructuración adversarial del campo político desplegada durante el debate de la cadena Globo, exponemos dos fragmentos adicionales que ilustran la operatoria analizada hasta aquí. En ambos, los debatientes se posicionan en un eje en cuyo opuesto aúnan a dos figuras; puntualmente, Boulos y Haddad confrontan con Temer y Bolsonaro. Ambos se apoyan sobre este eje para manifestar su oposición respecto de otros contrincantes en el piso. En el caso de Haddad, por último, la estrategia permite reforzar el acuerdo con su interlocutor, Boulos, a quien el candidato del PT halaga ("sos un candidato serio"), y con quien asume compartida una preocupación en torno a los candidatos que buscan "recortar derechos".

Alckmin (...), usted, junto con Temer, con Bolsonaro, apoyaron una reforma laboral que recortó derechos históricos de los trabajadores. (G. Boulos, G)

Boulos, le escogí porque usted es un candidato serio. Y yo quería preguntarle lo siguiente: hay tres candidatos que apoyan al gobierno de Temer, Henrique Meirelles, Geraldo Alckmin y quien es diputado hace 28 años, Jair Bolsonaro. Sólo hablan de recortar derechos (...) (F. Haddad, G).

Sintetizando lo expuesto hasta aquí, encontramos que en el encuentro organizado por la cadena Globo, al igual que en Argentina Debate, las figuras ausentes son de peso en la estructuración de la polémica. De manera igualmente afín al caso argentino, es más, las referencias a terceros que no están presentes en el estudio sirven de sustento para ciertas muestras de acuerdo entre los pretendidos contendientes. No obstante, hallamos diferencias entre los debates que nos ocupan. La trama confrontativa brasileña es más compleja. Los debatientes aúnan presentes y ausentes en polos polémicos diversos a partir de los cuales delimitan su propia posición. Las referencias a los ausentes no ofician de mero soporte al mutuo acuerdo, sino, también, edifican críticas entre contendientes. En este estudio de televisión, no todo es amistosidad. Con base en estas observaciones, derivaremos en lo que sigue nuestras conclusiones.

\section{Conclusiones}

Hemos dedicado la primera parte de este artículo a ensayar hipótesis que permitan comprender las inasistencias de J. Bolsonaro y D. Scioli a los debates presidenciales televisados inmediatamente anteriores a la primera vuelta de las elecciones. Con este fin, hemos considerado pertinente imbricar las ausencias en su contexto más amplio: el entorno político, con foco en la carrera electoral; el mediático, haciendo énfasis en la tradición de los debates a nivel nacional; y el de la trayectoria discursiva y comunicacional de cada candidato. Nuestra evidencia sugiere la potencial relevancia del criterio que advierte que 
los favoritos en la carrera electoral enfrentan mayores riesgos que beneficios al asistir a un debate presidencial. Puesto al revés, parece que los aspirantes a presidente sólo se arriesgan a ausentarse de un debate preelectoral si cuentan con el respaldo de una popularidad suficiente. A la decisión final pueden contribuir otros incentivos. También, experiencias pasadas que reduzcan la percepción de los costos de faltar. En cualquier caso, parece ser necesario imbuir a la inasistencia en una narrativa discursiva y un conjunto de prácticas comunicacionales que la justifiquen, le den sentido, y la hagan viable.

En la segunda parte, hemos examinado los efectos de las respectivas ausencias sobre el despliegue discursivo de las emisiones. Hemos encontrado, no obstante, cierta ambigüedad que el enunciador institucional en ambos debates pretende una posición neutral respecto de la actitud de los inasistentes. En contraste, hallamos que la ausencia adquiere un lugar central en la estructuración adversarial puesta de manifiesto en las argumentaciones de los debatientes. Hemos identificado ciertas lógicas polémicas similares, pero, también, importantes diferencias. De la comparación, extraemos a modo de cierre algunas conclusiones.

En primer lugar, destacamos el lugar privilegiado que cobra la ausencia en la confrontación. No sólo la propia falta, acción o actitud, es instrumentada como evidencia para derivar críticas hacia quienes rechazaron la propuesta de debatir en televisión; estrategia argumentativa que parece ser independiente de que la ausencia sea o no invocada a nivel de la puesta en escena o del formato. Sino, también, notablemente, la posición de contradestinatario es rellenada de manera reiterada por figuras externas, ajenas al intercambio entre los presentes en el piso, terceras.

En esta línea, en segundo lugar, hemos analizado que la referencia a terceros (ausentes) sirve para posicionar a los propios interlocutores, primeras y segundas personas entre las que se desenvuelve el diálogo. En el caso argentino, la operatoria es lineal y homogénea entre todos los debatientes: "nosotros, los presentes" versus "ellos, el kirchnerismo". En el caso brasileño, la trama de interrelaciones es más compleja. De todos modos, hemos observado que los diversos contendientes dan forma a la propia posición por referencia a las figuras que están fuera del estudio de televisión; sea por asociación, como es el caso de Haddad y Lula, sea por diferencia, como vimos para los candidatos que buscan recortar sus propias figuras sobre el trasfondo de las duplas del PT y de Bolsonaro, de Temer y Bolsonaro, o de Temer y el PT, o de estos tres en simultáneo. Asimismo, atendimos a la manera en que los debatientes brasileños colocan a sus destinatarios directos, presentes en el piso, en el lugar de la contradestinación por asociación con alguna de estas figuras ausentes.

De estas dinámicas de asociación-diferenciación, en tercer lugar, extrajimos una operación argumentativa de relevancia, común a las dos emisiones de debate que examinamos aquí. Esta consiste en la búsqueda de complicidad con el interlocutor a partir de la presuposición de la existencia de premisas compartidas, que se anclan en las figuras ausentes. Así, además de alimentar la polémica en el debate, el carácter tercero de la ausencia cooperó con el objetivo de promover el consenso, el acuerdo, y el refuerzo de propias y mutuas posiciones.

Hasta aquí, hemos sistematizado similitudes que hallamos entre las lógicas confrontativas desplegadas por los participantes de ambos debates. De manera complementaria, las di- 
ferencias entre los casos argentino y brasileño iluminan aspectos de las figuras ausentes, pero invocadas, que podemos conectar con lo elaborado en la primera parte de este trabajo, para ofrecer una lectura de conjunto.

Corriendo el foco del despliegue discursivo hacia los criterios estratégicos, la inasistencia de un candidato a un debate presidencial es una actitud fácilmente objetable por los presentes en el piso. Más aún, como aconteció en el caso argentino, si el formato y la puesta en escena invitan a entablar esta polémica a partir de la inscripción del silencio del ausente, de su vacío corporal. Ahora bien, la falta, por muy criticada, no aparenta ser el criterio determinante para la designación de un adversario privilegiado. Concretamente, no obstante las diversas tramas, hallamos que los terceros que los debatientes invocan comparten ciertas características. Se trata alternativamente de quienes estaban primeros en las encuestas, o quienes ejercían o habían ejercido de manera reciente cargos en la administración pública. En breve, parece que los ausentes deben encarnar de manera verosímil, hacer cuerpo, alternativamente, la representación de un mal reciente, o de un peligro inminente. Se desprende, adicionalmente, la conveniencia para los presentes de confrontar con quien no puede responder ni replicar. La combinación de estas razones parece explicar el diferente grado de complejidad que hallamos entre las tramas adversariales argentina y brasilera.

En cualquier caso, esta dinámica parece confirmar los peores temores del inasistente. Mientras nuestra evidencia parece confirmar las potenciales desventajas, sino peligros, que ofrece un debate presidencial televisado a quienes llevan la delantera en la carrera electoral y a los candidatos oficialistas, encuentra también que la inasistencia no evita que los candidatos sean tomados como objeto de la polémica. Es más: lo promueve. La evasiva deviene en profecía autocumplida.

En otro orden, la operatoria reformula de manera paradójica las aspiraciones de los debates presidenciales televisados: la confrontación in situ es opacada por una polémica desplazada, corrida. En el peor de los casos, los intercambios en el piso devienen un diálogo amistoso, como vimos que ocurrió en el primer encuentro organizado en la Argentina. Las consecuencias ulteriores que se desprendan de esta dinámica estratégica y argumentativa exceden los límites de este escrito. Presumimos que el despliegue confrontativo durante un debate presidencial televisado no es determinante de la repercusión mediática, mucho menos la político-electoral, que le prosigan. En todo caso, invitamos a producir investigaciones que hagan dialogar las dimensiones estratégicas y discursivas de los debates presidenciales televisados que abordamos aquí con otros corpus, contextos y fenómenos. También, a comparar las operatorias argumentativas entre estos y otros debates "incompletos", en los que un candidato invitado decide faltar, y encuentros en los que todos los convocados están presentes. Finalmente, sugerimos indagar el lugar del paradestinatario, tercera figura enunciativa clave en el discurso político, en el seno de estas dinámicas de polémica desplazada. En síntesis, queda por descubrir el efecto persuasivo que se deriva de la constitución de pro y contradestinatarios con apoyo de los ausentes en el piso de los debates presidenciales televisados. 


\section{Notas}

1. Recomendamos al lector interesado consultar los siguientes enlaces, que ofrecen información agregada de acuerdo con lo publicado por diversas encuestadoras a lo largo del tiempo. Para Brasil: https://es.wikipedia.org/wiki/Anexo:Sondeos_de_las_elecciones_presidenciales_de_Brasil_de_2018; para Argentina: https://es.wikipedia.org/wiki/ Anexo:Encuestas_de_intenci\%C3\%B3n_de_voto_para_las_elecciones_presidenciales_ de_Argentina_de_2015. La información primaria fue igualmente difundida por medios y por las propias encuestadoras en su momento. Por motivos de espacio no podemos extendernos en reponerla aquí.

2. Sobre el carácter de "indicador práctico" que poseen los resultados publicados de las encuestas preelectorales, recomendamos el trabajo de Vommaro (2008)

3. Fuente de este ejemplo: https://www.pagina12.com.ar/diario/

elpais/1-282462-2015-09-25.html

4. Otros medios, de tendencia más crítica hacia el gobierno de entonces, eran menos entusiastas a la hora de comunicar los resultados, y comenzaban a instalar la posibilidad de un ballotage. Con todo, el liderazgo de Scioli en las encuestas estaba fuera de discusión.

5. Un ejemplo entre muchos posibles: https:/extra.globo.com/noticias/brasil/datafolhabolsonaro-sobe-quatro-pontos-chega-32-haddad-tem-21-23121002.html

6. Nuestra interpretación se ve favorecida desde una lectura diacrónica de las estrategias de los respectivos candidatos. En efecto, ambos revierten sus posiciones vis-a-vis los debates presidenciales en distintos momentos de la campaña electoral, en correspondencia con la opinión pública relativa a los resultados esperados.

Para el kirchnerismo en general, y D. Scioli en particular, no descontamos el "efecto de lectura" tras las primera vuelta sobre la decisión de asistir al segundo debate presidencial televisado, que se realizó de manera previa al ballotage. A este efecto acompañó cierta interpretación mediática en torno a la inminencia de la "derrota". Por ejemplo, así comenzaba una nota de infobae: "Los resultados del 25 de octubre estuvieron lejos de lo que ansiaba el kirchnerismo: no sólo no consiguió imponerse en la primera vuelta, sino que terminó con un margen mucho más exiguo que el peor pronóstico que le daban las encuestas" (https://www.infobae.com/2015/11/03/1767109-el-escrutinio-definitivo-confirmo-el-ballotage-y-estiro-la-diferencia-daniel-scioli-y-mauricio-macri/).

En Jair Bolsonaro, la trayectoria es inversa: el candidato asiste a los primeros debates, y comienza a ausentarse en el momento en que su popularidad va in crescendo. Con todo, para comprender en detalle la situación del candidato brasileño debemos añadir la importante incidencia de otros factores: la prohibición de la candidatura de su principal competidor, I. Lula da Silva, y el atentado que sufrió el propio Bolsonaro en un acto de campaña. Ambos hechos acontecieron a comienzos de septiembre y marcan el punto inflexión a partir del cual el candidato comienza a ausentarse a los debates televisados.

7. En nuestro caso, hacemos foco en un sentido amplio en la incumbencia del partido, esto es, a las ventajas que posee el partido que busca reelección, antes que a la estricta incumbencia del candidato.

8. Desde los primeros estudios enfocados en cargos legislativos en Estados Unidos (Alford y Brady, 1989; Cover y Mayhew, 1977; Gelman y King, 1990; Jacobson y Carson, 
2015), se ha extendido el examen del efecto positivo de la incumbencia a las elecciones en otras latitudes. Mientras que en el primer caso la evidencia en favor de la ventaja de quien disputa la reelección es contundente, en otras regiones el efecto parece variar de acuerdo a otros factores, como sean la consolidación del sistema de partidos, o el contexto político más amplio (por ejemplo, Klašnja y Titiunik, 2017. La referencia ha sido extraída de Battocchio, 2018). De todos modos, a nuestros fines, basta hacer hincapié en la plausibilidad de que una suerte de creencia compartida relativa a las ventajas del oficialismo haya influido en la decisión de desestimar la invitación a debatir.

9. Si bien destacamos la alta fragmentación de este último (Mainwaring \& Shugart, 2018). 10. El concepto de institucionalización reúne diversas acepciones. Recomendamos la sistematización de Levitsky (2009. A nuestros fines, y dado el carácter exploratorio de nuestra empresa, resulta productivo de momento retener el término en su amplitud, para abarcar los diversos aspectos que hacen a la consolidación de los debates presidenciales televisados a nivel nacional.

11. Con todo, algunos autores indican que hay indicios de esta estrategia de enfrentamiento, o de cierta distancia, de manera previa a ese momento (Vincent, 2011; Fernández, 2014).

12. En el primer caso hacemos referencia a las transformaciones del ecosistema mediático impulsadas desde la legislación y la política pública. En el segundo, a la tendencia del kirchnerismo a eludir las mediaciones del periodismo, y optar por dispositivos de comunicación alternativos, con la pretensión de entablar un contacto "directo" con el electorado. Por ejemplo, se han examinado los discursos de atril de N. Kirchner (Dagatti, 2015), o el uso frecuente y distintivo de la Cadena Nacional por parte de la presidenta Cristina F. de Kirchner (Fernández, 2104; Cingolani, 2012). En tercer lugar, se ha explorado la relación adversativa que el discurso kirchnerista plantea con los medios de comunicación.

13. Fuente de la referencia: https://www.clarin.com/politica/elecciones_2015-

debate-scioli-chamuyo-macri_0_Sy7vBGtvmg.html

14. Fuente de este ejemplo: http://www.infobae.com/2015/09/23/1757395-daniel-sciolino-participara-del-debate-presidencial/

15. Recordamos, en línea con lo antes expuesto, que el incidente coincide temporalmente con la prohibición de la candidatura de Lula da Silva, y con un salto en la intención de voto hacia Bolsonaro.

16. Resulta interesante mencionar que el argumento intervino a nivel de la propia puesta en escena de la entrevista que ofreció Bolsonaro. A pesar de estar claramente editada, se incorporó la mostración de un médico que controlaba el bienestar del entonces candidato con cierta periodicidad. Puede accederse a la entrevista completa a partir del siguiente enlace: https://www.youtube.com/watch?v=2YnLFUiGF-I

17. Es decir, se mantuvo el esquema que había sido definido y sorteado entre equipos de campaña y realizadores del debate antes de que Scioli decidiera ausentarse. Puede consultarse el Manual de Estilo, resultado de estas negociaciones, en el siguiente enlace: http:// argentinadebate.org/wp-content/uploads/2015/09/ARGENTINA-DEBATE-2015-MANUAL-de-ESTILO.pdf

18. Los extractos que incorporamos para ilustrar nuestro análisis corresponden al desgravado de ambos debates. La referencia indica el nombre de quien pronunció las pa- 
labras, y la sigla "AD" o la letra "G" para referirnos alternativamente al encuentro organizado por Argentina Debate el día 4 de octubre de 2015, o al realizado por Globo el 4 de octubre de 2018. En el primer caso realizamos un desgravado manual a partir del registro audiovisual de la emisión (a la que se puede acceder en: https://www.youtube. com/watch?v=bkK8iI2qZVg). En el segundo caso, se trata de la traducción propia del desgravado que ofrece el siguiente enlace: https:/g1.globo.com/politica/eleicoes/2018/ noticia/2018/10/05/veja-a-integra-do-debate-na-globo.ghtml

Sólo en los casos en los que transcribimos un intercambio introduciremos los fragmentos mediante guión de diálogo (-).

19. Más aún, hemos examinado en otro lugar la estricta delimitación de los comentarios que los periodistas proferen a título personal, en primera persona, y su desempeño en el rol institucional, que lleva la voz del impersonal, durante el desarrollo del debate argentino. Este contrapunto no estuvo excento de tensiones (Franco, en prensa).

20. La única excepción es N. del Caño, que tiende a oponer un "nosotros, el FIT" a todos los demás (lo que supone colocar en el lugar de adversarios a D. Scioli junto con demás políticos en carrera).

\section{Bibliografía}

Acosta, M. (2016). La sociedad civil y el primer debate presidencial en Argentina. Marco. Revista de Marketing y Comunicación Política 2, pp. 111-130.

Alford, J. R. y Brady, D. W. (1989). Personal and Partisan Advantage in US Congressional Elections. En L. C. Dodd y B. I. Oppenheimer (Eds.), Congress Reconsidered 4th edition. Washington, DC: CQ Press.

Anderson, Perry. (2019). “Bolsonaro's Brazil”, London Review of Books 41(3). Disponible en: https://www.lrb.co.uk/v41/n03/perry-anderson/bolsonaros-brazil

Battocchio, M. E. (2018) La ventaja del oficialismo en las elecciones municipales de la provincia de Buenos Aires. Tesis de Maestría. Disponible en: http://sedici.unlp.edu.ar/ handle/10915/68720, https://doi.org/10.35537/10915/68720

Becerra, M. (23 de abril de 2008) Los medios salen del placard. Página/12. Disponible en: https://www.pagina12.com.ar/diario/elpais/1-102891-2008-04-23.html

Bennett, W. L. (2005) News. The Politics of Illusion. New York: Longman.

Cingolani, G (2012) "A midiatização da figura presidencial: espaços, estratégias e transições". En: Fausto Neto, A.; Mouchon, J. y Verón, E., Transformações de midiatização presidencial: corpos, relatos, negociações, resistências. São Paulo: Difusão Editores.

Cover, A. D. y Mayhew, D. R. (1977). Congressional Dynamics and the Decline of Competitive Congressional Elections. En L. C. Dodd y B. I. Oppenheimer (Eds.), Congress Reconsidered. New York: Praege

Dagatti, M. (2015). Los discursos de atril de Néstor Kirchner, o cómo construir liderazgo en sociedades mediatizadas. Disponible en: http://semioticadelosmedios.sociales.uba.ar/

De Diego, J. (2014). “¿Discurso político o politicidad de los discursos? Una propuesta para pensar la relación entre kirchnerismo y prensa”. En Lis Gindin, I. (coord.), Kirchnerismo, 
mediatización e identidades políticas: reflexiones en torno a la política, el periodismo y el discurso. 2003-2008; 1a ed. - Rosario: UNR Editora. Editorial de la Universidad Nacional de Rosario. E-Book.

Fernández, M. (2014). "Periodismo y política en la Argentina kirchnerista: disputas por la intermediación en el espacio público. Un análisis desde la perspectiva de la mediatización”. En: Lis Gindin, I. (coord.), Kirchnerismo, mediatización e identidades políticas: reflexiones en torno a la política, el periodismo y el discurso. 2003-2008; 1a ed. - Rosario: UNR Editora. Editorial de la Universidad Nacional de Rosario. E-Book.

Franco, C. (en prensa) "Argentina Debate 2015. El debate presidencial televisado en el centro de los debates". En: Dagatti y Aimá (coomps.) Hegemonía discursiva e identidades políticas en la Argentina contemporánea. Buenos Aires: Teseo.

Gelman, A. y King, G. (1990). Estimating Incumbency Advantage Without Bias. American Journal of Political Science 34, pp. 1142-1164.

Hallin, D. y Mancini, P. (2004), Comparing Media Systems. Three Models of Media and Politics. Cambridge: Cambridge UP.

Jacobson, G. y Carson, J. (2015). The Politics of Congressional Elections. Maryland: Rowman \& Littlefield Publishers.

Jamieson, K.H. y Birdsell, D.S. (1988). Presidential Debates. The challenge of creating an informed electorate. New York Oxford: Oxford University Press.

Katz, E y Dayan, D. (1992). Media Events: the live broadcasting of history. Cambridge: Harvard University Press.

Klašnja, M. y Titiunik, R. (2017). The Incumbency Curse: Weak Parties, Term Limits, and Unfulfilled Accountability. American Political Science Review, 111(01), 129-148.

Kitzberger, P. (2011). "La madre de todas las batallas. El kirchnerismo y los medios de comunicación”. En: Malamud, A. y De Luca, M. (coords.). La política en los tiempos de Kirchner. Buenos Aires: Eudeba.

Kitzberger, P. (2016). "Media Wars and the New Left: Governability and Media Democratisation in Argentina and Brazil", Journal of Latin American Studies 48 (3).

Levitsky, S. (2009)- "Institutionalization: Unpacking the Concept and Explaining Party Change" en D. Collier and J. Gerring (eds). Concept and Methods in Social Science. Routledge.

Mainwaring, S., \& Shugart, M. (2018). Presidencialismo y sistema de partidos en América Latina. Revista Uruguaya De Ciencia Política, (9), 9-40. Disponible en: http://rucp.cienciassociales.edu.uy/index.php/rucp/article/view/393

Meyhew, L.H. (1997) The New Public. Professional Communication and the Means of Social Influence. Cambridge: Cambridge UP.

Norris, P. (2000). A virtuous circle. Political Communications in Post-industrial Societies. Cambridge: Cambridge UP.

Quirós, L. (2015). ¿La silla vacía? Debates presidenciales en América Latina. Disponible en: https://www.opendemocracy.net/democraciaabierta/ludmila-quir\%C3\%B3s/danielscioli-y-las-\%E2\%80\%9Csillas-vac\%C3\%ADas\%E2\%80\%9D-en-am\%C3\%A9rica-latina

Raiter, A. (2013) “¿Existe una lógica discursiva kirchnerista? Constancias y alternancias”. En: Balsa, J. (comp.) Discurso, política y acumulación en el kirchnerismo. Buenos Aires: Ediciones del CCC, UNQ. 
Slimovich, A. (2012). El debate electoral en los viejos y nuevos medios. Entre entimemas y emociones. En: RILL Nueva época, Prácticas discursivas a través de las disciplinas 17 (1/2), INSIL, UNT. Disponible en: http://www.insil.com.ar/adminis/up-load/upload/ rill2012/Art\%C3\%ADculo\%2019\%20SLIMOVICH,\%20Ana.pdf

Verón, E. (1993) La semiosis social. Fragmentos de una teoría de la discursividad. Barcelona: Gedisa.

Verón, E. (17 de enero de 2014). Reflexiones sobre la ausencia. El liberal. Disponible en: http://www.elliberal.com.ar/noticia/121935/reflexiones-sobre-ausencia

Vommaro, G. (2008). "Lo que quiere la gente": los sondeos de opinión y el espacio de la comunicación política en Argentina (1983-1999). Buenos Aires: Prometeo.

Waisbord, S. (1995). El gran desfile. Campañas electorales y medios de comunicación en la Argentina. Buenos Aires: Sudamericana.

\begin{abstract}
Organized by the NGO Argentina Debate, the first Argentinian presidential debate was held on October the 4th, 2015. The incumbent, Daniel Scioli, had announced he would skip this debate the week before: an unexpected resolution. An empty chair was left where his place would have been. Exactly three years later, on October the 4th, 2018, the last debate prior to the first round of Brazil's presidential elections was held, part of an already well-established tradition. Using his heath as an excuse, candidate Jair Bolsonaro skipped this televised meeting.

The object of this article consists of this two "missings". Its point of departure is that not going to a presidential debate is a costly decision for a presidential candidate. The article first explores hypothesis that can explain the decision to skip the debate in terms of its tactical implications. Second, the article turns to observe the absence as a cause, examining its discursive effects. The analysis is situated on two levels: strategic and discursive. The conclusions hang upon this twofold analysis, while taking advantage of a comparative approach.
\end{abstract}

Keywords: presidential debates - political speech - electoral campaigns.

Resumo: No dia 4 de outubro do 2015 ocorreu o primeiro debate presidencial na Argentina, organizado pela ONG Argentina Debate. O candidato oficialista, Daniel Scioli, decidiu se ausentar uma semana antes da data agendada para a realização do debate, uma escolha inesperada que teve como resultado a forte imagem de um lugar vazio durante a transmissão. Exatamente 3 anos depois, 4 de otubro de 2018 o então candidato Jair Bolsonaro não assistiu ao encontro prévio a primera ronda de comissões presidenciais no Brasil, parte de uma tradição de debates já consolidada no país.

Partindo da base de que não assistir a um debate presidencial televisionado é uma decisão cara para um candidato na carreira presidencial, o presente artigo tem por objeto analisar ambas ausências. Em primeiro lugar, explora hipóteses que explicam a ausência em termos de uma opção estratégica. Ou seja, como conseqüência de um certo equilíbrio entre 
custos e benefícios dentro de uma série de condições. Isso está fundamentado em literatura pré-existente e em fontes secundárias. Em segundo lugar, examina os efeitos discursivos que a ausência produz durante o desenvolvimento das transmissões de debates. A análise está em dois níveis. Por um lado, se questiona brevemente como a falta apresentada pelos organizadores do debate, no nível da organização e no formato das trocas. Por outro, permite o exame do papel da ausência para a estruturação contraditória do campo político (Verón, 1987) nos argumentos dos debatedores. As conclusões conectam as duas perspectivas, estratégicas e discursivas, e os resultados oferecidos pela abordagem comparativa.

Palavras-chave: Debates presidenciais na televisão - discurso político - campanhas eleitorais.

[Las traducciones de los abstracts fueron supervisadas por el autor de cada artículo] 